\title{
The One-Two Punch of Delirium and Dementia During the COVID-19 Pandemic and Beyond
}

\author{
Sara C. LaHue ${ }^{1,2 *}$, Vanja C. Douglas ${ }^{1,2}$ and Bruce L. Miller ${ }^{1,2}$ \\ ${ }^{1}$ Department of Neurology, School of Medicine, University of California, San Francisco, San Francisco, CA, United States, \\ ${ }^{2}$ Department of Neurology, Weill Institute for Neurosciences, University of California, San Francisco, San Francisco, CA, \\ United States
}

Keywords: delirium, dementia, Alzheimer's disease and related dementias (ADRD), COVID-19, hospitalization, accessibiity, technology

\section{INTRODUCTION}

While the severe acute respiratory syndrome coronavirus 2 (SARS-CoV-2) pandemic is an unprecedented threat to all of us, older adults are especially at risk for serious complications from coronavirus disease 2019 (COVID-19) that necessitate hospitalization $(1,2)$. Significant attention has rightfully been given to the respiratory and cardiovascular consequences of COVID-19. Less is understood about the neurologic complications associated with this virus, especially its potential impact on delirium and cognitive decline.

The prevalence of delirium is expected to increase during this pandemic due to several factors detailed below (3). Delirium is independently associated with accelerated cognitive decline for those with and without preexisting dementia (4). Adults with Alzheimer's disease and related dementias (ADRD) are particularly vulnerable during this pandemic due to dependence on others and increased likelihood of living in assisted living facilities (5). Further, 30\% of all COVID-19 deaths in the United States occur in patients living in nursing homes; a high percentage of these individuals also suffer from mild cognitive impairment or dementia (6). If hospitalized, those with ADRD are more likely to become delirious (7). Our goals are to highlight the heightened risk of this neurologic "one-two punch" as well as provide pragmatic, evidence-based management recommendations for a several clinical environments during the COVID-19 pandemic and subsequent recovery period.

\section{RECOGNIZING THE HIDDEN DELIRIUM EPIDEMIC WITHIN THE PANDEMIC}

This article was submitted to Dementia and Neurodegenerative

Diseases,

a section of the journal Frontiers in Neurology

Received: 18 August 2020

Accepted: 07 October 2020

Published: 05 November 2020

Citation:

LaHue SC, Douglas VC and Miller BL

(2020) The One-Two Punch of Delirium and Dementia During the COVID-19 Pandemic and Beyond.

Front. Neurol. 11:596218. doi: 10.3389/fneur.2020.596218

Delirium is a common acute disturbance in mental status characterized by fluctuations in attention and cognition, that is more common in older adults and those with dementia (4). Compared with non-delirious patients, hospitalized delirious patients are more likely to develop functional impairment, be discharged to a facility, and be readmitted to the hospital $(4,8)$. While there are established clinical pathway interventions that modify the risk of developing delirium, there is no drug currently recommended for the prevention or treatment of delirium other than medications needed to treat an identified provoker (e.g., antibiotics for infection) $(3,9)$. Rather, delirium management centers on non-pharmacologic measures, and the removal of psychoactive medications unless necessary for significant agitation (3). Identifying and managing patients with delirium is paramount as the ability to safely discharge patients home, which is necessary for sustaining strained healthcare systems with limited hospital resources during this pandemic.

While quantifying the impact of this pandemic on delirium is in its early stages, COVID-19 has been demonstrated in small studies to be associated with an increased risk of delirium $(10,11)$. This 
association is likely due to an exacerbation of established risk factors, including the development of hypoxia, metabolic derangements, and infection leading to a heightened inflammatory state, as well as the need for intensive care $(12,13)$. In one point-prevalence cohort study of 71 hospitalized individuals with COVID-19, 31 patients were diagnosed with delirium by DSM-IV criteria (11). Importantly, only 12 of these patients were recognized as being delirious by the primary clinical team (11). Such a discrepancy is not surprising since, without the use of a targeted screening tool, misidentification of delirium for depression or other psychiatric diagnoses, especially in older patients is not uncommon $(14,15)$. This underdiagnosis of delirium highlights the need for structured clinical pathways with systematic screening to identify delirium so as to appropriately manage these patients (9). Accurate delirium identification is also instrumental for providing informed projections of the long-term resources needed to support those who survive.

One critical outcome of this pandemic is the anticipated exacerbation of delirium in all hospitalized patients, regardless of COVID-19 status $(3,16)$. This is in large part due to challenges with implementing proper delirium prevention guidelines (3). For example, prior to this pandemic, many hospitals across the United States housed Acute Care for Elders (ACE) Units that incorporated evidence-based practices for delirium prevention spearheaded by the Hospital Elder Life Program (4). Such programs significantly reduced hospitalassociated complications for older adults, including delirium, which can be prevented in as many as $40 \%$ of cases with these modest interventions (4). However, the management of hospitalized adults has undergone significant systematic changes in response to COVID-19, regardless of COVID-19 status $(3,17)$. With many hospitals reaching or exceeding patient capacity during this pandemic, some ACE units have disbanded in order to reallocate resources for the care of patients with COVID-19, subsequently fracturing delirium prevention care pathways as well. Furthermore, hospitals have placed restrictions on visitors of hospitalized patients, some prohibiting all visitors regardless of COVID-19 status, with rare exceptions $(3,16,18)$. Such restrictions also often apply to caregivers for patients who are delirious or have ADRD, despite playing an essential role in patient care by encouraging physical and cognitive stimulation to prevent delirium, protecting their loved one from falls, and advocating for their basic needs, such as oral hydration $(3,9,16)$.

Delirium prevention is even more challenging for hospitalized adults with COVID-19. In addition to strict visitor restrictions, all patients hospitalized for COVID-19 experience heighted solitude and reduced physical activity from quarantine in order to reduce exposure to staff and preserve personal protective equipment (PPE) (16). Sleep may be disrupted if tests occur at night to ensure adequate time for equipment sterilization. While designed to minimize infection risk, these policies also foster a deliriogenic environment (3). Though these unfavorable environmental changes are new, this novel virus has largely incited a resurgence of traditional risk factors for hospital-acquired delirium, and in doing so has exposed fissures in the clinical care of these patients that require urgent, creative solutions.

\section{NOVEL CHALLENGES FOR ADULTS WITH ALZHEIMER'S DISEASE AND RELATED DEMENTIAS}

Adults with ADRD are especially vulnerable to COVID19 infection due to their older age, frequency of comorbid conditions such as cardiovascular disease and diabetes, increased reliance on assistance from others for survival, and a higher likelihood of living in assisted living or nursing home, making self-quarantine difficult for many $(5,19)$. Depending on the severity of the dementia or presence of associated behavioral symptoms, those with ADRD may have difficulty understanding or remembering public health recommendations, such as maintaining good hand hygiene, social distancing, and wearing a face covering (20). Further, in the event that support systems fail-if a family member becomes ill, grocery delivery is not possible, or medication refills delayed-additional harm can result (21). Combined, these challenges may lead to significant psychosocial stressors for those with ADRD and their families during this pandemic, which are only exacerbated in the event of hospitalization $(3,21-23)$.

Adults with ADRD, especially those diagnosed with COVID19 , are at heightened risk for both hospitalization and delirium during this pandemic $(7,24)$. While changes to hospital policies, such as restricted visitation and need for isolation, are important for infection control, the consequences of these guidelines are anticipated to be especially detrimental to this population. Even once delirium resolves, its effects can persist, as delirium is associated with an accelerated rate of cognitive decline in those with ADRD (7). Delirium is also an independent risk factor for new cognitive decline in those without pre-existing cognitive impairment (25). In one prospective study, 9.5\% of cognitively normal adults with post-operative delirium developed mild cognitive impairment or dementia within a year (25). In addition to the physical and psychological challenges that COVID-19 survivors face, a surge in delirium during this pandemic may lead to a delayed epidemic of cognitive impairment.

\section{ADAPTING EVIDENCE-BASED PRACTICES FOR DELIRIUM AND ADRD MANAGEMENT}

The tenets of delirium prevention are evidence-based and are not antithetical to the precautions needed to care for hospitalized patients, especially those with ADRD, during this pandemic (3). Rather, these recommendations can be adapted for patients with COVID-19, as well as those without COVID-19 but who are still significantly affected by changes in hospital and community policies (Table 1).

There are several novel strategies for coping with the isolation from social distancing, largely revolving around technological tools $(3,16)$. Ideally, the caregivers of hospitalized COVID19 negative patients with delirium or ADRD would be viewed as essential to patient care and allowed visitation, while still adhering to hospital policies on symptom screening and PPE. If this is not possible, then adapting hospital teleconferencing tools, such as providing electronic tablets, can enhance communication 
TABLE 1 | Adapting management practices for hospitalized patients with delirium or dementia during the COVID-19 pandemic.

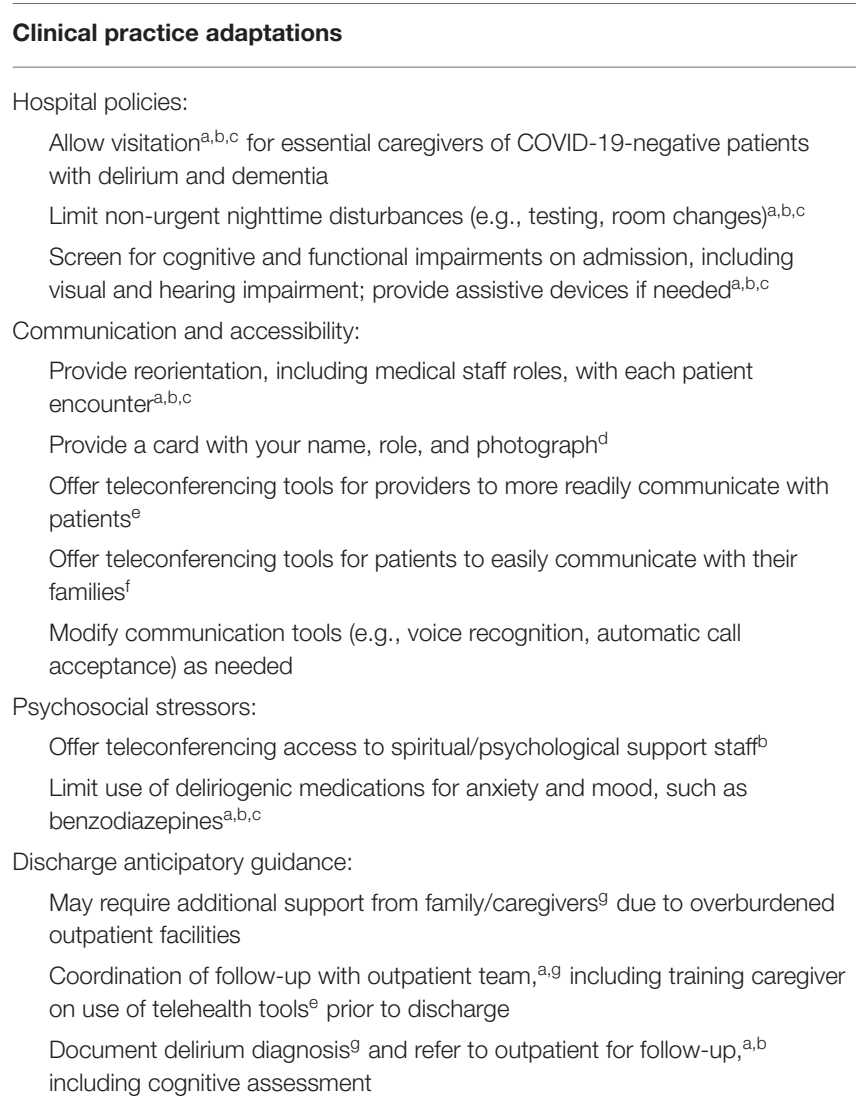

${ }^{a}$ Hshieh et al. (9).

${ }^{b}$ Inouye et al. (26).

${ }^{c}$ Wang et al. (27).

${ }^{d}$ Arora et al. (28).

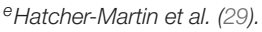

${ }^{f}$ Hart et al. (18).

gKhachaturian et al. (30).

between patients and their families (18). These tools can also increase access to nursing and spiritual/psychological support staff while minimizing infection risk and PPE use.

An important caveat that receives insufficient consideration is that many of these modifications assume abilities on the part of the patient. For instance, those with hearing impairment are at increased risk for delirium and should be provided with assistive devices, such as pocket amplification devices, in order to use teleconferencing tools $(9,26,27,31)$. Recognizing a patient's premorbid cognitive and functional status is important for providing accessible means of communication. Voice recognition software on smartphones and computers allows for placing calls without physically interacting with the device; however, this may not be feasible for individuals with delirium, advanced ADRD or language impairment. One innovative alternative for these individuals is to enable the automatic call accept feature found on many devices, permitting the device to automatically answer telephone calls, or video calls such as FaceTime, from known contacts. This exciting accessibility feature may facilitate more frequent patient contact with family and staff for those who have difficulty interfacing with digital tools. Employing technology creatively to reduce social isolation, provide cognitive stimulation, and more readily assess basic needs in patients with delirium and advanced ADRD is an area that warrants increased attention.

In addition to hospital-specific considerations, the management of adults with ADRD during this pandemic must span the entire continuum of care. Disruption to clinic appointments, even with telemedicine capabilities, will likely result in increased demand for care. Community outpatient access is vital for keeping those with ADRD supported at home and out of the hospital. The rapid expansion of access to teleconferencing tools, including the waiving of Health Insurance Portability and Accountability Act compliance in order to permit more popular video chat applications, is a valuable step (32). Clinical assessments must also be adapted to suit the constraints of digital technologies. For many medical specialties, including several neurologic subspecialities, there is a trend toward non-inferiority of remote assessments compared to in-person visits $(17,29)$. While it is possible to offer cognitive testing by telemedicine, accuracy may be affected by hearing and vision impairment and so the results of these tests must be interpreted these limitations in mind (33-35). In addition to the special considerations for patients with ADRD, adults diagnosed with hospital-associated delirium should receive outpatient follow-up, including cognitive assessments to screen for cognitive decline. Just as battling this pandemic has been resource-intensive, so too will providing adequate resources to support survivors long-term.

\section{CONCLUSIONS}

Delirium is a common complication faced by hospitalized older adults that can often be prevented with modest interventions. Unfortunately, the rate of delirium will likely increase during the COVID-19 pandemic due to both infectious and environmental considerations. While ADRD is a risk factor for delirium, delirium is also an independent risk factor for cognitive decline. If delirium prevention is neglected, and we fail to adapt clinical care to meet these unique challenges, we risk an unprecedented accelerated growth in cognitive impairment in the months and years to come. We cannot afford to let our guard down at this critical juncture.

\section{AUTHOR CONTRIBUTIONS}

SL made substantial contributions to conception or design of the work, drafted the work, gave final approval of the version to be published, and agreed to be accountable for all aspects of the work. VD and BM made substantial contributions to conception or design of the work, revised the work critically for important intellectual content, gave final approval of the version to be published, and agreed to be accountable for all aspects of the work. All authors contributed to the article and approved the submitted version. 


\section{REFERENCES}

1. Bianchetti A, Bellelli G, Guerini F, Marengoni A, Padovani A, Rozzini $\mathrm{R}$, et al. Improving the care of older patients during the COVID-19 pandemic. Aging Clin Exp Res. (2020) 32:1883-8. doi: 10.1007/s40520-02001641-W

2. Wu Z, Mcgoogan JM. Characteristics of and important lessons from the coronavirus disease 2019 (COVID-19) outbreak in China: summary of a report of 72314 cases from the Chinese Center for Disease Control and Prevention. JAMA. (2020) 323:1239-42. doi: 10.1001/jama. 2020.2648

3. Kotfis K, Williams Roberson S, Wilson JE, Dabrowski W, Pun BT, Ely EW. COVID-19: ICU delirium management during SARS-CoV-2 pandemic. Crit Care. (2020) 24:176. doi: 10.1186/s13054-020-02882-x

4. Inouye SK, Westendorp RG, Saczynski JS. Delirium in elderly people. Lancet. (2014) 383:911-22. doi: 10.1016/S0140-6736(13)60688-1

5. Wang $\mathrm{H}, \mathrm{Li} \mathrm{T}$, Barbarino $\mathrm{P}$, Gauthier S, Brodaty $\mathrm{H}$, Molinuevo JL, et al. Dementia care during COVID-19. Lancet. (2020) 395:1190-1. doi: 10.1016/S0140-6736(20)30755-8

6. Behrens LL, Naylor MD. "We are alone in this battle": a framework for a coordinated response to COVID-19 in nursing homes. J Aging Soc Policy. (2020) 32:316-22. doi: 10.1080/08959420.2020.1773190

7. Fong TG, Davis D, Growdon ME, Albuquerque A, Inouye SK. The interface between delirium and dementia in elderly adults. Lancet Neurol. (2015) 14:823-32. doi: 10.1016/S1474-4422(15)00101-5

8. Lahue SC, Douglas VC, Kuo T, Conell CA, Liu VX, Josephson SA, et al. Association between inpatient delirium and hospital readmission in patients $>/=65$ years of age: a retrospective cohort study. J Hosp Med. (2019) 14:201-6. doi: 10.12788/jhm.3130

9. Hshieh TT, Yang T, Gartaganis SL, Yue J, Inouye SK. Hospital elder life program: systematic review and meta-analysis of effectiveness. Am J Geriatr Psychiatry. (2018) 26:1015-33. doi: 10.1016/j.jagp.2018.06.007

10. Benussi A, Pilotto A, Premi E, Libri I, Giunta M, Agosti C, et al. Clinical characteristics and outcomes of inpatients with neurologic disease and COVID-19 in Brescia, Lombardy, Italy. Neurology. (2020) 95:e910-20. doi: 10.1212/WNL.0000000000009848

11. Mcloughlin BC, Miles A, Webb TE, Knopp P, Eyres C, Fabbri A, et al. Functional and cognitive outcomes after COVID-19 delirium. Eur Geriatr Med. (2020) 11:857-62. doi: 10.1007/s41999-020-00353-8

12. Fotuhi M, Mian A, Meysami S, Raji CA. Neurobiology of COVID-19. J Alzheimers Dis. (2020) 76:3-19. doi: 10.3233/JAD-200581

13. Paterson RW, Brown RL, Benjamin L, Nortley R, Wiethoff S, Bharucha T, et al. The emerging spectrum of COVID-19 neurology: clinical, radiological and laboratory findings. Brain. (2020) 143:3104-20. doi: 10.1093/brain/aw aa240

14. Farrell KR, Ganzini L. Misdiagnosing delirium as depression in medically ill elderly patients. Arch Intern Med. (1995) 155:2459-64.

15. Otani VHO, Otani T, Freirias A, Calfat ELB, Aoki PS, Cordeiro QJ, et al. Misidentification of mental health symptoms in presence of organic diseases and delirium during psychiatric liaison consulting. Int J Psychiatry Clin Pract. (2017) 21:215-20. doi: 10.1080/13651501.2017.1301483

16. Lahue SC, James TC, Newman JC, Esmaili AM, Ormseth CH, Ely EW. Collaborative delirium prevention in the age of COVID-19. J Am Geriatr Soc. (2020) 68:947-49. doi: 10.1111/jgs.16480

17. Chen PM, Hemmen TM. Evolving healthcare delivery in neurology during the coronavirus disease 2019 (COVID-19) pandemic. Front Neurol. (2020) 11:578. doi: 10.3389/fneur.2020.00578

18. Hart JL, Turnbull AE, Oppenheim IM, Courtright KR. Family-centered care during the COVID-19 era. J Pain Symptom Manage. (2020) 60:e93-7. doi: 10.1016/j.jpainsymman.2020.04.017

19. Iaboni A, Cockburn A, Marcil M, Rodrigues K, Marshall C, Garcia MA, et al. Achieving safe, effective, and compassionate quarantine or isolation of older adults with dementia in nursing homes. Am J Geriatr Psychiatry. (2020) 28:835-8. doi: 10.1016/j.jagp.2020.04.025

20. Brown EE, Kumar S, Rajji TK, Pollock BG, Mulsant BH. Anticipating and mitigating the impact of the COVID-19 pandemic on Alzheimer's disease and related dementias. Am J Geriatr Psychiatry. (2020) 28:712-21. doi: 10.1016/j.jagp.2020.04.010

21. Greenberg NE, Wallick A, Brown LM. Impact of COVID-19 pandemic restrictions on community-dwelling caregivers and persons with dementia. Psychol Trauma. (2020) 12:S220-1. doi: 10.1037/tra0000793

22. Boutoleau-Bretonniere C, Pouclet-Courtemanche H, Gillet A, Bernard A, Deruet AL, Gouraud I, et al. The effects of confinement on neuropsychiatric symptoms in Alzheimer's disease during the COVID-19 crisis. J Alzheimers Dis. (2020) 76:41-7. doi: 10.3233/JAD-200604

23. Brooks SK, Webster RK, Smith LE, Woodland L, Wessely S, Greenberg N, et al. The psychological impact of quarantine and how to reduce it: rapid review of the evidence. Lancet. (2020) 395:912-20. doi: 10.1016/S0140-6736(20)30460-8

24. Miyashita S, Yamada T, Mikami T, Miyashita H, Chopra N, Rizk D. Impact of dementia on clinical outcomes in elderly patients with coronavirus 2019 (COVID-19): an experience in New York. Geriatr Gerontol Int. (2020) 20:7324. doi: $10.1111 /$ ggi.13942

25. Sprung J, Roberts RO, Weingarten TN, Nunes Cavalcante A, Knopman DS, Petersen RC, et al. Postoperative delirium in elderly patients is associated with subsequent cognitive impairment. Br J Anaesth. (2017) 119:316-23. doi: $10.1093 /$ bja/aex130

26. Inouye SK, Bogardus ST Jr, Baker DI, Leo-Summers L, Cooney LM Jr. The hospital elder life program: a model of care to prevent cognitive and functional decline in older hospitalized patients. Hosp Elder Life Program J Am Geriatr Soc. (2000) 48:1697-706. doi: 10.1111/j.1532-5415.2000.tb03885.x

27. Wang YY, Yue JR, Xie DM, Carter P, Li QL, Gartaganis SL, et al. Effect of the tailored, family-involved hospital elder life program on postoperative delirium and function in older adults: a randomized clinical trial. JAMA Intern Med. (2019) 180:e194446. doi: 10.1001/jamainternmed.2019.4446

28. Arora V, Gangireddy S, Mehrotra A, Ginde R, Tormey M, Meltzer D. Ability of hospitalized patients to identify their in-hospital physicians. Arch Intern Med. (2009) 169:199-201. doi: 10.1001/archinternmed.2008.565

29. Hatcher-Martin JM, Adams JL, Anderson ER, Bove R, Burrus TM, Chehrenama $\mathrm{M}$, et al. Telemedicine in neurology: telemedicine work group of the American Academy of Neurology update. Neurology. (2020) 94:30-8. doi: 10.1212/WNL.0000000000008708

30. Khachaturian AS, Hayden KM, Devlin JW, Fleisher LA, Lock SL, Cunningham $\mathrm{C}$, et al. International drive to illuminate delirium: a developing public health blueprint for action. Alzheimers Dement. (2020) 16:711-25. doi: 10.1002/alz.12075

31. Lahue SC, Liu VX. Loud and clear: sensory impairment, delirium, and functional recovery in critical illness. Am J Respir Crit Care Med. (2016) 194:252-3. doi: 10.1164/rccm.201602-0372ED

32. Padala SP, Jendro AM, Orr LC. Facetime to reduce behavioral problems in a nursing home resident with Alzheimer's dementia during COVID-19. Psychiatry Res. (2020) 288:113028. doi: 10.1016/j.psychres.2020.113028

33. Brearly TW, Shura RD, Martindale SL, Lazowski RA, Luxton DD, Shenal BV, et al. Neuropsychological test administration by videoconference: a systematic review and meta-analysis. Neuropsychol Rev. (2017) 27:174-86. doi: 10.1007/s11065-017-9349-1

34. Hantke NC, Gould C. Examining older adult cognitive status in the time of COVID-19. J Am Geriatr Soc. (2020) 68:1387-9. doi: 10.1111/jgs.16514

35. Phillips NA, Chertkow H, Pichora-Fuller MK, Wittich W. Special issues on using the montreal cognitive assessment for telemedicine assessment during COVID-19. J Am Geriatr Soc. (2020) 68:942-4. doi: 10.1111/jgs.16469

Conflict of Interest: The authors declare that the research was conducted in the absence of any commercial or financial relationships that could be construed as a potential conflict of interest.

Copyright (c) 2020 LaHue, Douglas and Miller. This is an open-access article distributed under the terms of the Creative Commons Attribution License (CC BY). The use, distribution or reproduction in other forums is permitted, provided the original author(s) and the copyright owner(s) are credited and that the original publication in this journal is cited, in accordance with accepted academic practice. No use, distribution or reproduction is permitted which does not comply with these terms. 